\title{
Threshold anomaly in non-central forces
}

\author{
J. Gómez-Camacho and M.A. Nagarajan ' \\ Depariamento de Fisica tiómica. Itolecular y Nuclear. Inwersidad de Sevilla. tpdo 1065. E-41080 Sevilla. Spain
}

Received 9 November 1992

\begin{abstract}
The behaviour of the threshold anomaly for non-central potentials, which account for collective excitations in heavy-ion collisions. is investigated. It is shown that the non-central potentuals should exhibit an energy dependence at energies in the vicinity of the Coulomb barrier This energy dependence is. however. different from that of the elastic optical potential. occurring at lower energies It if further shown that there are corrections to the traditional collective model such that. If the transition potential is expressed as the derivative of the optical potential, the corresponding deformation length will be complex and energy-dependent Simple model calculations are presented
\end{abstract}

Durıng recent years, evidence of the energy-dependence of the real (the threshold anomaly) and imaginary parts of nucleus-nucleus optical potentials has been found by careful analyses of the elastic scattering at energies in the vicinity of the Coulomb barrier [1-5] This energy dependence has been attributed to the coupling of non-clastic channels to the elastic channel in this energy region [6.7]. The threshold anomaly has also been related to the rapid increase of the surface imaginary potential as the energy is increased above the Coulomb barrier and the consequent correction to the real potential through a dispersion relation [8-10]. The optical potential can thus be written as

$l^{\prime}(E)=V_{0}+\Delta l^{\prime}(E)+{ }_{1} W^{\prime}(E)$.

where $V_{0}$ is an energy-independent potential (double-folded potential, for example). $W^{\circ}(E)$ is the imaginary potential and the dispersive real potential $\Delta I^{\prime}(E)$ is defined as

$\Delta V^{\prime}(E)=\frac{\mathrm{P}}{\pi} \int \frac{W^{\prime}\left(E^{\prime}\right)}{E^{\prime}-E^{\prime}} \mathrm{d} E^{\prime}$.

where $\mathrm{P}$ denotes a principal value integral.

Generalızıng this to the case of a set of strong-coupled channels, it was suggested by Satchler [11] that

1 Permanent address SERC Daresbury Laboratory. Daresbury, Warrington WA4 4.AD, UK each element of the potential matrix, which defines the coupled channels equation. should be expected to exhibit a threshold anomaly. In such a case, the energy dependence of the different diagonal and off-diagonal elements of the potential matrix will be attributed to couphings to other channels not included in the subset of coupled channels. However. a priorı. there is no reason for all the terms of the potential matrix to have the same energy dependence. Satchler [11] argued that, in the case of collective excitations, one would expect a similar energy dependence in the coupling interaction (transition potential) and the elastic optical potential, though one could expect a shift in the threshold of the coupling potential by approximately half of the excitation energy. He further concludes that if one uses an optical potential that is consistent with the dispersion relation but still needs an energy-dependent deformation length, then either the optical model itself or the nuclear structure model. or both, are inadequate

The excitation functions of the first excited $3^{-}$state of ${ }^{208} \mathrm{~Pb}$ by ${ }^{16} \mathrm{O}$ at energies around the Coulomb barrier have been measured and analysed [12,13] and more recently detalled angular distributions for the same system were measured at energies from above to below the Coulomb barrier [14]. These angular distributions were analyzed and the results hinted at a possibility that the transition potential may have an 
energy dependence which is different from that of the elastic optical potential [14].

We present here a simple model which shows that. for collective excitations, the central and couplıng potentials. although consistent separately with dispersion relations, could exhibit different energy dependences Note that coupling potentials include the case of reorientation potentials for the case of elastic scaltering of nucles with $I \geqslant 1$ Let us consider the usual collective model description of inclastic excitation. The optical potential $C^{\prime}$ is considered as a function of the collective variable $\delta$, which is a measure of the change in distance of the surfaces of the colliding nucle. The real and imaginary parts of the potential can be expanded in a Taylor series in powers of $\delta$. and to the first order in $\delta$.

$I(\delta)=I-\frac{\mathrm{d} I}{\mathrm{~d} r} \delta . \quad H(\delta)=U-\frac{\mathrm{d} H}{\mathrm{~d} r} \delta$.

If the potentials are exponentual in the region of interest. then

$\mathrm{I}^{\prime}(\delta)=\mathrm{I}^{\prime}(1+\delta / a), \quad \boldsymbol{H}^{\prime}(\delta)=\boldsymbol{H}^{\prime}(1+\delta / a)$.

where $a$ is the diffuseness parameter, which has been assumed to be the same for the real and imaginary potentials. Note that, when expressing the imaginary potential as a function of $\delta$. we are implicilly assumIng that the collective variable changes slowly. com. pared with the processes that contribute to the imaginary potential That implies also that the energy of the barrier $B$ will be a function of $\delta$. Approximately. we can write

$B(\delta) \simeq B(1-\delta / R)$.

where $B$ is the height of the undeformed barrier and $R$ is the corresponding radius. To explore the implications of the effect of $\delta$ on both the geometry of the potential as well as on the barrier, we consider a factorizable form of $H^{\circ}(E)$

$W(E)=W F(x)$.

where $I$ is a function of the radius while $F(x)$ expresses the energy dependence in terms of the parameter $x=(B-E) / .1 . F(x)$ is assumed to be a Ferm function $F(x)=[1+\exp (x)]^{-1}$. The parameter 1 is a measure of the energy range over which the imaginary potential increases. According to eqs (4) and
(5). both $H$ and $F(x)$ are dependent on the collecuve variable $\delta$.

With the ansatz for $H^{\circ}(E)$ of eq. $(6)$. the dispersive potential $\Delta l(E)$ of eq. (1) becomes

$\Delta I^{\prime}(E)=W G(x)$.

where $G(x)$ is related to $F(x)$ through the dispersion relation

$G(x)=\frac{\mathrm{P}}{\pi} \int_{-\infty}^{x} \frac{F(y)}{y-x} \mathrm{~d} y$.

The optical potential can be written as

$$
l^{\prime}(E, \delta)=I^{\prime}(E . \delta)+1 \omega(E \delta \delta) .
$$

To first order in $\delta$. one obtains

$$
\begin{aligned}
& I(E, \delta)=I_{0}+H(i(x) \\
& +\frac{\delta}{a}\left[1+W G(x)+\| \epsilon\left(g^{\prime}(x)\right]\right. \text {. } \\
& \Psi(E, \delta)=W F(x) \\
& +\frac{\dot{\delta}}{a}\left[H F(x)+W_{\epsilon} F^{\prime}(x)\right] .
\end{aligned}
$$

where we have introduced the parameter $\epsilon=a B / R A$ and used eq. (4). In these equations, the diagonal potential $L_{0}(E)$ is the term independent on $\delta$ and the transition potentual $\zeta_{1}(E)$ is the term linear in $\delta$. We can write this explicitly as

$$
\begin{aligned}
& L_{0}(E)=I_{0}+H(F(x)+1 H F(x) . \\
& l_{1}(E) \\
& \quad=-\delta\left(\frac{\mathrm{d} l_{0}(E)}{\mathrm{d} r}+\frac{\epsilon}{a} W^{\prime}\left(G^{\prime}(x)+{ }_{1} F^{\prime}(x)\right)\right) .
\end{aligned}
$$

The first term on the right of eq. (13) is the usual prescription of the collectuve model where the transition potential is related to the derivative of the central potential. The new feature of this model is the second term on the right which arises from the shift of the barrier due to deformation. Eqs (12) and (13) suggest that, while the central terms will exhibit the threshold anomaly around an energy $B$, the non-central terms will exhıbit it around an energy $B(1-a)$ $R$ ). Besides, all the non-central terms will exhibit the threshold anomaly at the same energy. independently of the multipolarity or the coupling strength 
(provided that a first order treatment is valid)

It is of interest to point out that exactly the same expressions (12) and (13) are obtained for the central and transition potentials if one considers the adiabatıc lımit when the coupled equations can be decoupled into eigenchannels. In the case of including the ground state and a one-phonon state, the two ergenchannels describe the scattering by potentuals of $\operatorname{rad}_{11} R \pm \delta$, with the corresponding barriers $B(1 \mp \delta /$ $R)$. The transition potential is half the difference of the two eigenchannel potentials and up to order $\delta$ yelds exactly eq. (13).

If one still defines the coupling potential by

$U_{\mathrm{t}}(E)=-\delta_{\mathrm{eff}} \frac{\mathrm{d} L^{\prime}(E)}{\mathrm{d} r}$,

the ratio of the effective to the static deformation length of the potential becomes complex and energy dependent:

$R(E)=\frac{\delta_{\mathrm{eff}}}{\delta}=1-\epsilon \frac{G^{\prime}(x)+1 F^{\prime}(x)}{V_{0} / W^{\prime}+G(x)+1 F(x)}$.

Eq. (15) shows separately the contributions to the effective deformation of the static deformation and of the dispersive effect due to the change in the barrier energies. $R(E)$ acquires an imaginary part in the vicinity of the barrier. of the order of $\epsilon H^{\prime} / 4 I(B)$. The real part of $R(E)$ has an energy dependence around the barrier that is consistent with the imaginary part through dispersion relations.

The size of the correction to the conventional model depends crucially on the parameter $\epsilon$, that is determined primarily by the range $A$ over which the imaginary potential rises (for $E=B-A$, the imaginary potential is $27 \%$, while for $E=B+1$, it is $73 \%$ of the maxımum value). Depending on the value of $\epsilon$, one obtains different shapes of the energy dependence of the imaginary part of the transition potential. For $\epsilon<1$, the imaginary transition potential rises monotonically to a constant value $\delta \mathrm{d} \omega \% \mathrm{~d} r$. For $\epsilon>1$. one observes a characteristic maximum in the imaginary transition potential just above the barrier, and then a gradual decrease to the value $\delta \mathrm{d} W / \mathrm{d} r$ at larger energies. We illustrate this in figs. 1 and 2 , where we show both the elastic (central) and transition potentials for the inelastic scattering of ${ }^{16} \mathrm{O}$ on ${ }^{208} \mathrm{~Pb}$ to the first $3^{-}$ state of the target. Calculations have been done taking $B=80 \mathrm{MeV}, a=06 \mathrm{fm}$ and $R=12 \mathrm{fm}$. Two val-

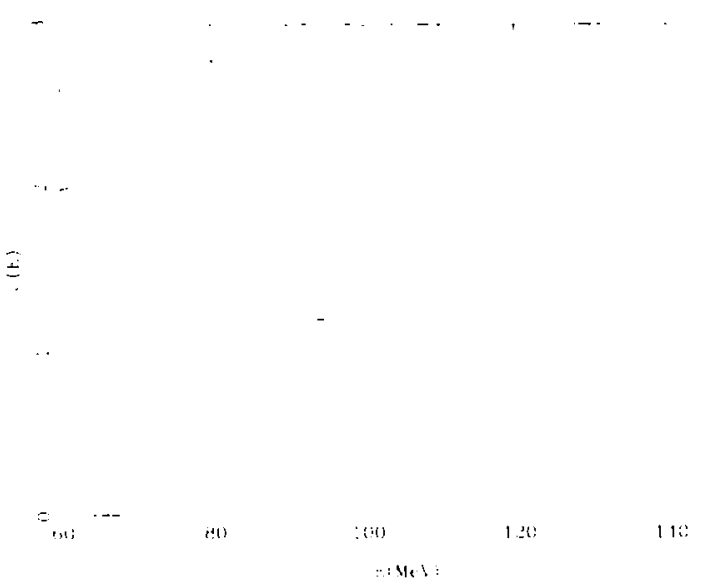

Fig I Central (full line) and non-central (dashed line) potentuals versus the energy for $J=25 \mathrm{MeV}$ The real parts are the upper lines. and the imaginary parts are the lower lines

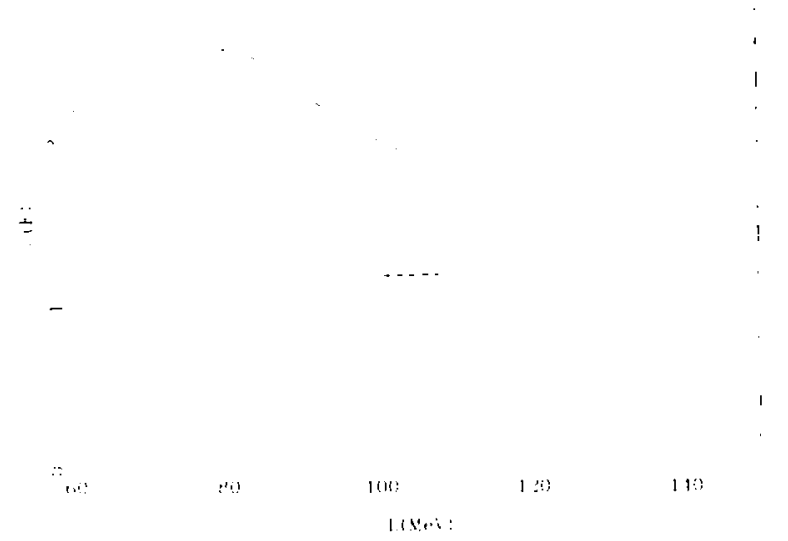

Fig 2 Same as fig 1 for $J=455 \mathrm{MeV}$.

ues of $J$. which are $J=4.55 \mathrm{MeV}$, taken from ref. [9], and $J=2.50 \mathrm{MeV}$. have been used. In both cases. the optical potential is taken as $1.5+11.2 \mathrm{McV}$ for $E=150$ $\mathrm{MeV}$. Both calculations are consistent with the empirical data of the optical potential of ${ }^{16} \mathrm{O}+{ }^{208} \mathrm{~Pb}$. These yield values of $\epsilon$ of 088 and 1.6 respectively. The transition potential is normalized so that it coincides with the central potential at high energies. It is clearly seen that the energy dependence of the transition and central potentials are different in an energy region around the Coulomb barrier. Even though from figs. 1 and 2 it may appear that if $\epsilon<1$, the traditional collectuve model, where the transition poten- 
thal is taken as the product of a static deformation length and the derivative of the complex potential, is valid. this is not strictly true In fig. 3 we show the real and imaginary parts of $R(E)$ as a function of the energy for the two cases $.1=4.55 \mathrm{MeV}$ and $J=2.5$ $\mathrm{MeV}$ It is seen that even for $1=4.55 \mathrm{MeV}(\epsilon<1)$. the deformation length becomes complex. It is evident. of course, that very high quality inelastic data will be necessary to extract this complex deformation length at energies around the Coulomb barrier

The parameter $\epsilon$ can be understood in a time-dependent picture. $\tau=h / .1$ has been described as a retardation time in the tume-dependent picture of the imaginary potential [ 15 ]. It is the characteristic tume for the processes that contribute to the imaginary potential, that is, for the coupling to states not related to the collective variable $\delta . a B / R$ is approximately equal to $\mathrm{I}$ Classically. the hamiltonian for a vibratuonal collecuve variable $\delta$ is

$I=P_{\delta}^{2} / 2 I I+K^{2} \delta^{2} / 2-I^{\prime} \exp (\delta / a)$.

where $M$ and $K$ are. respectuvely, the inerta parameter and the harmonic constant of the collective vartable We can define the characteristic time for collectuve excitation as

$1 / T=\frac{\mathrm{d} P_{\delta} / \mathrm{d} t}{P_{s}}$
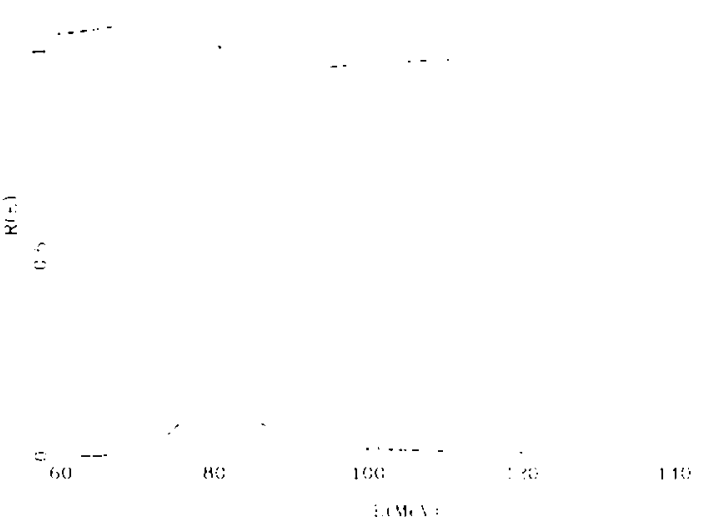

Fig 3 Real (upper lines) and imaginary (lower lines) parts of the ratio of the effective and static deformation lengths as a function of the energy The full lines correspond to $1=25 \mathrm{MeV}$ and the dashed lines to $1=455 \mathrm{McV}$ $\mathrm{d} P_{o} / \mathrm{d} t$ can be obtained from eq. (16) using Hamilton's equation

$\frac{\mathrm{d} P_{\delta}}{\mathrm{d} t}=-\frac{\mathrm{d} H}{\mathrm{~d} \delta}=-\frac{\delta}{C}+\frac{\square}{a} \exp (\delta / a)$.

The second term on the RHS dominates over the first in the adiabatıc lımıt. On the other hand. as $\delta$ will take values of the order of $a$, we can estımate the value of $P_{o}$ as $\hbar / a$. Thus, $T=\hbar R / a B$ is the characteristic time for collective excitations. Hence the parameter $\epsilon=\tau / T$ is the ratio of the tume for processes that take away flux from the coupled channels system to the time for the processes that couple within the coupled channels system If $\tau<T$. the coupling to non-collective channels is fast compared to the coupling to collectuve channels. and then its effect on the diagonal and transition potentials is similar, producing a simlar energy dependence of both. If $\tau>T$, the coupling to non-collective channels is slow compared to that of collectuve channels. and so a different energy dependence appears. Note that these conditions are not incompatible with the assumption that the collective variable changes slowly compared with processes that contribute to the imaginary potential. The characteristic tıme of change of $\dot{\delta}$ can be defined as

$1 / T=\frac{\mathrm{d} \delta / \mathrm{d} t}{\delta}$

$\mathrm{d} \delta / \mathrm{d} t$ can be obtained from eq. (16) using Hamılton's equation

$\frac{\mathrm{d} \delta}{\mathrm{d} t}=\frac{\mathrm{d} M}{\mathrm{~d} P} P_{b}=\frac{P_{i}}{M}$.

Hence. using the previous estumates for $\delta$ and $P_{\delta}$, one gets $T^{\prime \prime}=1 / a^{2} / \hbar$, which can be larger than $\tau$ if the inertia parameter is large enough.

To conclude. we have shown that the transition potentuals for collective excitation have a different energy dependence to the diagonal potentials. The deformation length of the potential becomes complex and energy-dependent around the Coulomb barrier. The size of this effect depends crucially on how fast the imaginary optical potential rises to its maximum value as the energy increases. These cffects could be investigated measuring the scattering of polarized projectiles at energies around the Coulomb barrier. 
J.G.C. acknowledges partial support from the Spanish DGICYT under contract PB89-0636. M.A.N wishes to thank the Spanısh DGICYT for enabling a sabbatıcal stay at the University of Sevilla.

\section{References}

[1] A. Baeza, B Bılwes, R Bilwes. J Diaz and J L Ferrero, Nucl Phys A 419 (1984) 412 .

[2] J S Lilley, B R Fulton, M A Nagarajan, $1 \mathrm{~J}$ Thompson and D.W Barnes, Phys Lett B 162 (1985) 55

[3] B R Fulton, D W Barnes, J S. Lilley, M A Nagarajan and I J. Thompson Phys. Lett. B 162 (1985) 55

[4] B Bilwes. R Bilwes. J Diaz and J.L Ferrero. Nucl Phys A 449 (1986) 519

[5] A M. Stefanını et al, Phys Rev Lett 59 (1987) 2852
[6] I J Thompson, M A Nagarajan, J S Lilley and B R Fulton, Phys. Lett B 157 ( 1985 ) 250

[7] S C. Pleper, M J Rhoades-Brown and S Landowne, Phys Lett B $162(1985) 43$

[8] M A Nagarajan, C Mahaux and G R Satchler, Phys Rev Lett 54 (1985) 1136

[9] C Mahaux, G R Satchler and H Ngo, Nucl. Phys. A 449 (1986) 354

[10] G R. Satchler, Phys Rep 199 (1991) 147.

[11] G R. Satchler, Nucl Phys A 472 (1987) 591

[12] S Landowne, CH Dasso and G Pollarolo, Phys Lett B 178 (1986) 336, B 223 (1986) 277

[13] C S Lim, M D Belgetz. R H Spcar, W J. Vermeer, M P Fewell. B A Robson and R Smıth. Phys Lett. B 200 ( 1988) 263

[14] M J Smıthson. J S Lilley, M A Nagarajan, P V Drumm. R A Cunningham. B R Fulton and I J Thompson. Nucl Phys A 517 (1990) 183

[15] C Mahaux, K TR Davies and GR Satchler, preprin ULG-PNT-92-3-P (1992), Phys Rep (1993), to appear 